\title{
Long Strength of Layered Composite under Normal Fracture
}

\author{
Vladimir I. Andreev ${ }^{1,}$, Robert A. Turusov², b and Nikita Yu. Tsybin ${ }^{3, c}$ \\ 1,2,3 Moscow State University of Civil Engineering, Yaroslavskoye sh., 26, \\ Moscow 129337 Russia \\ a $\underline{\text { asv@mgsu.ru, }},{ }^{\text {rob-turusov@yandex.ru, }}{ }^{\mathrm{C}}$ nikitacybin@gmail.com
}

Keywords: composite, creep, contact layer, long strength

\begin{abstract}
The article deals with the stress-strain state of layered composite of rectangular section under tension by distributed uniform normal load $q$. Interaction between layers is accomplished by the contact layer in which is mixed the substances of adhesive and the substrate.
\end{abstract}

\section{Introduction}

Using the contact layer in the solution of the problem allows us to overcome problems such as endless tangential stresses arising at the border between the layers and allows to calculate the physical properties of the contact layer on the basis of experimental data.

We will consider the contact layer as the transversal anisotropic medium with such parameters that it can be represented as a set of short elastic rods, not connected among themselves. For simplicity, we assume that the rods are oriented normal to the contact surface.

\section{Statement of the Problem}

Fig. 1 shows a square element with any two layers $(k-1$ and $k$ ). Rigid connection between layers is accomplished by the contact layer, in which is mixed the substances of the two layers.

We assume that in each layer of the composite in addition to elastic deformations present forced (temperature), and high-elastic deformations:

$$
\varepsilon_{i, k}=\varepsilon_{i, k}^{e}+\varepsilon_{i, k}^{t}+\varepsilon_{i, k}^{h . \mathrm{e}},
$$

where $i=x, y, z ; k$ - layer number; $\varepsilon_{i, k}^{e}$ - elastic, $\varepsilon_{i, k}^{t}$ - forced, $\varepsilon_{i, k}^{h . e .}-$ high-elastic deformations. Components $\varepsilon_{i, k}^{t}$ and $\varepsilon_{i, k}^{h . e .}$ are defined by follows expressions:

$$
\varepsilon_{i, k}^{t}=\alpha_{i, k} \Delta T_{i, k} ; \quad \varepsilon_{i, k}^{h . \mathrm{e} .}=\sum_{s=1}^{2} \varepsilon_{s, i, k}^{h . e .}
$$

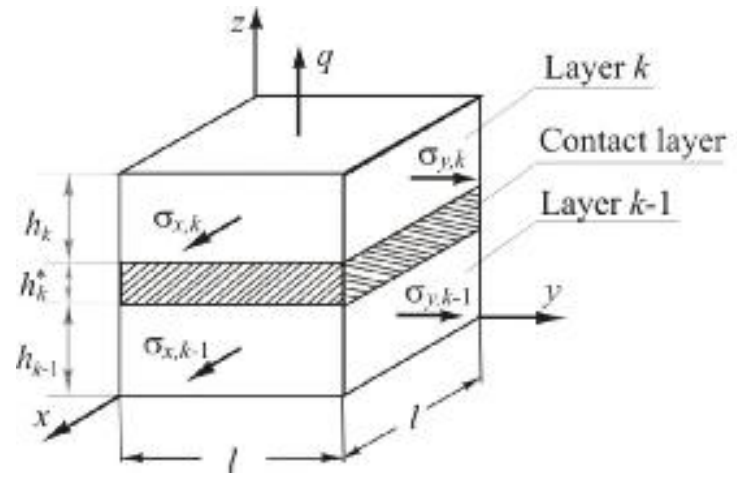

Figure 1. Model of a layered composite

Two summands $(s=1,2)$ in the second Eq. (1) correspond to two components of the relaxation time spectrum and are determined by the expressions [1]:

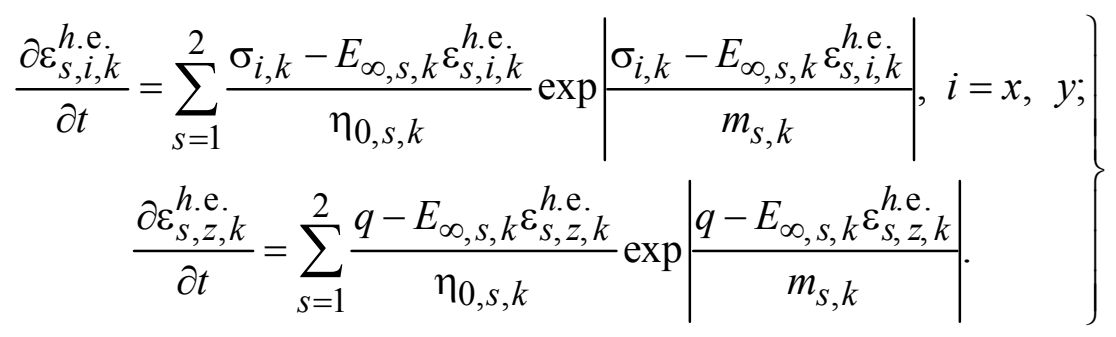




\section{Derivation of Resolving Equations}

Assuming that the contact layer consists of a plurality of short elastic rods, it perceives only the

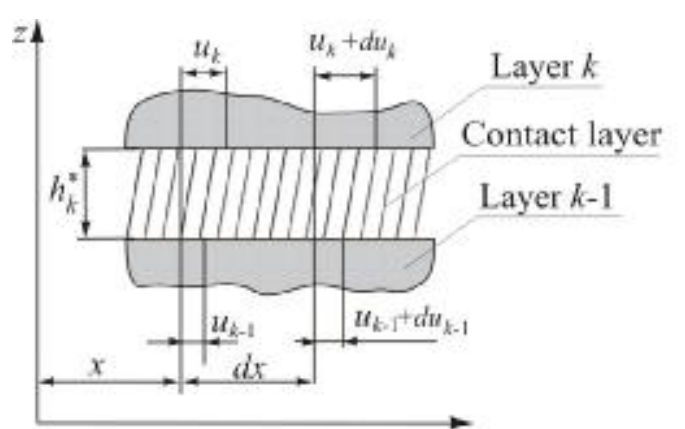

Figure 2. Displacements in the contact layer

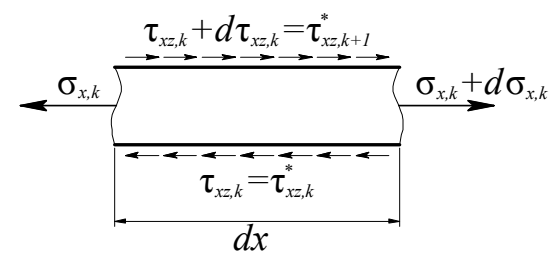

Figure 3. Equilibrium of the layer

tangential stresses $\tau_{z x, k}^{*}, \tau_{z y, k}^{*}$, normal stresses $\sigma_{z, k}^{*}=q$ and normal stresses $\sigma_{x, k}^{*}=\sigma_{y, k}^{*}=0$. Here and further, all values marked with * relate to the contact layer. Since the rods are short, the displacement $u$ and $v$ into the contact layer (Fig. 2) can be regarded as linear functions of the coordinate $z$ :

$$
u_{k}^{*}=a_{k}^{*}+b_{k}^{*} z ; \quad v_{k}^{*}=c_{k}^{*}+d_{k}^{*} z
$$

Since bending is missing, displacement in the contact layer are functions of the variable $z: w_{k}^{*}=w_{k}^{*}(z)$. In view of this, shear deformations of the contact layer will take the form:

$$
\begin{aligned}
& \gamma_{x z, k}^{*}=\frac{\partial u_{k}^{*}}{\partial z}+\frac{\partial w_{k}^{*}}{\partial x}=\frac{\partial u_{k}^{*}}{\partial z}=b_{k}^{*} \\
& \gamma_{y z, k}^{*}=\frac{\partial v_{k}^{*}}{\partial z}+\frac{\partial w_{k}^{*}}{\partial y}=\frac{\partial v_{k}^{*}}{\partial z}=d_{k}^{*} .
\end{aligned}
$$

By virtue of the continuity of displacements $u$ and $v$ on the surface of the contact of layers in accordance with (4), we obtain

$$
\gamma_{x z, k}^{*}=b_{k}^{*}=\frac{u_{k}-u_{k-1}}{h_{k}^{*}} ; \quad \gamma_{y z, k}^{*}=d_{k}^{*}=\frac{v_{k}-v_{k-1}}{h_{k}^{*}}
$$

Also in accordance with the hypothesis of thin plates, we assume that stresses in each layer does not depend of the variable $z$. Then, according to Fig. 3, the equilibrium conditions of any $k$-th layer can be written as

$$
\tau_{x z, k}^{*}-\tau_{x z, k+1}^{*}=h_{k} \frac{\partial \sigma_{x, k}}{\partial x} ; \quad \tau_{y z, k}^{*}-\tau_{y z, k+1}^{*}=h_{k} \frac{\partial \sigma_{y, k}}{\partial y} .
$$

For the material of the contact layer Hooke's law has the form

$$
\gamma_{x z, k}^{*}=\frac{\tau_{x z, k}^{*}}{G_{k}^{*}} ; \gamma_{y z, k}^{*}=\frac{\tau_{y z, k}^{*}}{G_{k}^{*}},
$$

from which it follows

$$
\tau_{x z, k}^{*}=\frac{G_{k}^{*}}{h_{k}^{*}}\left(u_{k}-u_{k-1}\right) ; \quad \tau_{y z, k}^{*}=\frac{G_{k}^{*}}{h_{k}^{*}}\left(v_{k}-v_{k-1}\right) .
$$

Let us differentiate the first Eq. in (8) with respect to $x$ and second Eq. with respect to $y$ :

$$
\frac{\partial \tau_{x z, k}^{*}}{\partial x}=\frac{G_{k}^{*}}{h_{k}^{*}}\left(\frac{\partial u_{k}}{\partial x}-\frac{\partial u_{k-1}}{\partial x}\right) ; \quad \frac{\partial \tau_{y z, k}^{*}}{\partial x}=\frac{G_{k}^{*}}{h_{k}^{*}}\left(\frac{\partial v_{k}}{\partial x}-\frac{\partial v_{k-1}}{\partial x}\right) .
$$

Next, using the Duhamel physical Eqs. and Cauchy relations, we obtain 


$$
\left.\begin{array}{rl}
\frac{\partial \tau_{x z, k}^{*}}{\partial x}= & \frac{G_{k}^{*}}{h_{k}^{*}}\left(\varepsilon_{x, k}-\varepsilon_{x, k-1}\right)=\frac{G_{k}^{*}}{h_{k}^{*}}\left[\frac{1}{E_{k}}\left(\sigma_{x, k}-\mu_{k} q-\mu_{k} \sigma_{y, k}\right)+\varepsilon_{x, k}^{t}+\varepsilon_{x, k}^{h . \text {.e }}\right]- \\
& -\frac{G_{k}^{*}}{h_{k}^{*}}\left[\frac{1}{E_{k-1}}\left(\sigma_{x, k-1}-\mu_{k-1} q-\mu_{k-1} \sigma_{y, k-1}\right)+\varepsilon_{x, k-1}^{t}+\varepsilon_{x, k-1}^{h . \text {.e. }}\right] ; \\
\frac{\partial \tau_{y z, k}^{*}}{\partial x}= & \frac{G_{k}^{*}}{h_{k}^{*}}\left(\varepsilon_{y, k}-\varepsilon_{y, k-1}\right)=\frac{G_{k}^{*}}{h_{k}^{*}}\left[\frac{1}{E_{k}}\left(\sigma_{y, k}-\mu_{k} q-\mu_{k} \sigma_{x, k}\right)+\varepsilon_{y, k}^{t}+\varepsilon_{y, k}^{h . \text {.e }}\right]- \\
& -\frac{G_{k}^{*}}{h_{k}^{*}}\left[\frac{1}{E_{k-1}}\left(\sigma_{y, k-1}-\mu_{k-1} q-\mu_{k-1} \sigma_{x, k-1}\right)+\varepsilon_{y, k-1}^{t}+\varepsilon_{y, k-1}^{h . \text {.e. }}\right] .
\end{array}\right\}
$$

Let us differentiate the first Eq. in (6) with respect to $x$ and second Eq. with respect to $y$ :

$$
h_{k} \frac{\partial^{2} \sigma_{x, k}}{\partial x^{2}}=\frac{\partial \tau_{x z, k}^{*}}{\partial x}-\frac{\partial \tau_{x z, k+1}^{*}}{\partial x} ; \quad h_{k} \frac{\partial^{2} \sigma_{y, k}}{\partial y^{2}}=\frac{\partial \tau_{y z, k}^{*}}{\partial y}-\frac{\partial \tau_{y z, k+1}^{*}}{\partial y} \text {. }
$$

Substituting (10) into (11) and introducing the notation $g_{k}^{*}=G_{k}^{*} / h_{k}^{*}$, we obtain resolving Eqs.:

$$
\begin{aligned}
& h_{k} \frac{\partial^{2} \sigma_{x, k}}{\partial x^{2}}=g_{k}^{*}\left\{\frac{1}{E_{k}}\left[\sigma_{x, k}-\mu_{k}\left(q+\sigma_{y, k}\right)\right]-\frac{1}{E_{k-1}}\left[\sigma_{x, k-1}-\mu_{k-1}\left(q+\sigma_{y, k-1}\right)\right]+\varepsilon_{x, k}^{t}+\varepsilon_{x, k}^{h . e .}-\varepsilon_{x, k-1}^{t}-\varepsilon_{x, k-1}^{h . e .}\right\}- \\
& -g_{k+1}^{*}\left\{\frac{1}{E_{k+1}}\left[\sigma_{x, k+1}-\mu_{k+1}\left(q+\sigma_{y, k+1}\right)\right]+\varepsilon_{x, k+1}^{t}+\varepsilon_{x, k+1}^{h . e .}\right\}+g_{k+1}^{*}\left\{\frac{1}{E_{k}}\left[\sigma_{x, k}-\mu_{k}\left(q-\sigma_{y, k}\right)\right]+\varepsilon_{x, k}^{t}+\varepsilon_{x, k}^{h . e .}\right\} ; \\
& h_{k} \frac{\partial^{2} \sigma_{y, k}}{\partial y^{2}}=g_{k}^{*}\left\{\frac{1}{E_{k}}\left[\sigma_{y, k}-\mu_{k}\left(q+\sigma_{x, k}\right)\right]-\frac{1}{E_{k-1}}\left[\sigma_{y, k-1}-\mu_{k-1}\left(q+\sigma_{x, k-1}\right)\right]+\varepsilon_{y, k}^{t}+\varepsilon_{y, k}^{h . e .}-\varepsilon_{y, k-1}^{t}-\varepsilon_{y, k-1}^{h . e .}\right\}- \\
& -g_{k+1}^{*}\left\{\frac{1}{E_{k+1}}\left[\sigma_{y, k+1}-\mu_{k+1}\left(q+\sigma_{x, k+1}\right)\right]+\varepsilon_{y, k+1}^{t}+\varepsilon_{y, k+1}^{h . e .}\right\}+g_{k+1}^{*}\left\{\frac{1}{E_{k}}\left[\sigma_{y, k}-\mu_{k}\left(q+\sigma_{x, k}\right)\right]+\varepsilon_{y, k}^{t}+\varepsilon_{y, k}^{h . e}\right\} .
\end{aligned}
$$

\section{Example of Calculation}

We consider three-layer composite consisting of the two identical substrate layers bonded with adhesive (fig. 4). For simplicity, we will consider sample with square cross-section with dimensions $l \times l$.

By virtue of the fact that the substrate layers are identical, we have

$$
\begin{aligned}
& E_{0}=E_{2} ; \mu_{0}=\mu_{2} ; \alpha_{0}=\alpha_{2} ; h_{0}=h_{2} ; \\
& h_{1}^{*}=h_{2}^{*} ; G_{1}^{*}=G_{2}^{*} ; g_{1}^{*}=g_{2}^{*} .
\end{aligned}
$$

From the equilibrium conditions for the composite follows

$$
\sigma_{x, 0} h_{0}=\sigma_{x, 2} h_{2}=-\frac{\sigma_{x, 1} h_{1}}{2} .
$$

Because the cross-section is square, in Eq. (12) must take into account the fact that the laws of the distribution of the stresses $\sigma_{x, k}$

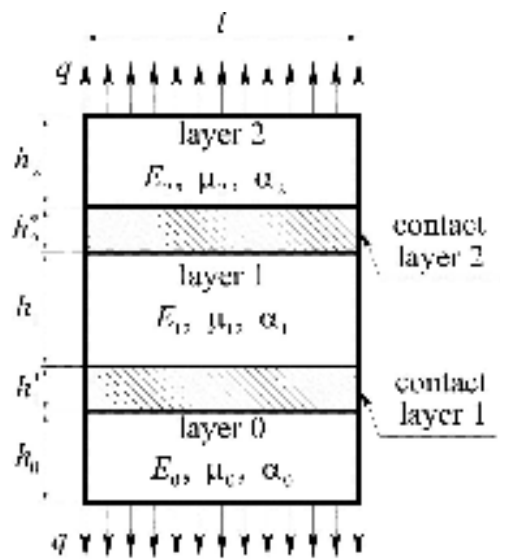

Fig. 4 The calculation scheme and $\sigma_{y, k}$ are equal. This implies

$$
\begin{gathered}
h_{k} \frac{\partial^{2} \sigma_{x, k}}{\partial x^{2}}=g_{k}^{*}\left\{\frac{1}{E_{k}}\left[\sigma_{x, k}\left(1-\mu_{k}\right)-\mu_{k} q\right]+\varepsilon_{x, k}^{t}+\varepsilon_{x, k}^{h . e .}\right\}-g_{k}^{*}\left\{\frac{1}{E_{k-1}}\left[\sigma_{x, k-1}\left(1-\mu_{k-1}\right)-\mu_{k-1} q\right]+\varepsilon_{x, k-1}^{t}+\varepsilon_{x, k-1}^{h . e .}\right\}- \\
-g_{k+1}^{*}\left\{\frac{1}{E_{k+1}}\left[\sigma_{x, k+1}\left(1-\mu_{k+1}\right)-\mu_{k+1} q\right]+\varepsilon_{x, k+1}^{t}+\varepsilon_{x, k+1}^{h . e .}\right\}+g_{k+1}^{*}\left\{\frac{1}{E_{k}}\left[\sigma_{x, k}\left(1-\mu_{k}\right)-\mu_{k} q\right]+\varepsilon_{x, k}^{t}+\varepsilon_{x, k}^{h . e .}\right\} .
\end{gathered}
$$

From the condition [1] $\varepsilon_{x, s}^{h . \text { e. }}+\varepsilon_{y, s}^{h . \text { e. }}+\varepsilon_{z, s}^{h . \text { e. }}=0, s=1,2$ we get 


$$
\varepsilon_{x, k}^{h . e .}=-\frac{1}{2} \varepsilon_{z, k}^{h . e .}
$$

Using equalities (1) and (16) from (15) we obtain for $k=1$ :

$$
\begin{aligned}
h_{1} \frac{\partial^{2} \sigma_{x, 1}}{\partial x^{2}} & =\left\{\frac{1}{E_{1}}\left[\sigma_{x, 1}\left(1-\mu_{1}\right)-\mu_{1} q\right]+\left(\alpha_{1} \Delta T-\frac{1}{2} \varepsilon_{z, 1}^{\text {h.e. }}\right)\right\}\left(g_{1}^{*}+g_{2}^{*}\right)- \\
& -g_{1}^{*}\left\{\frac{1}{E_{0}}\left[\sigma_{x, 0}\left(1-\mu_{0}\right)-\mu_{0} q\right]+\left(\alpha_{0} \Delta T-\frac{1}{2} \varepsilon_{z, 0}^{\text {h.e. }}\right)\right\}- \\
& -g_{2}^{*}\left\{\frac{1}{E_{2}}\left[\sigma_{x, 2}\left(1-\mu_{2}\right)-\mu_{2} q\right]+\left(\alpha_{2} \Delta T-\frac{1}{2} \varepsilon_{z, 2}^{\text {h.e. }}\right)\right\} .
\end{aligned}
$$

Given (13) and (14), and assuming that the high-elastic deformations of the substrate are zero, this expression can be transformed into

$$
\frac{1}{2} \frac{h_{1}}{g_{1}^{*}} \frac{\partial^{2} \sigma_{x, 1}}{\partial x^{2}}=\sigma_{x, 1}\left[\frac{\left(1-\mu_{1}\right)}{E_{1}}+\frac{h_{1}}{2 h_{0}} \frac{\left(1-\mu_{0}\right)}{E_{0}}\right]+q\left(\frac{\mu_{0}}{E_{0}}-\frac{\mu_{1}}{E_{1}}\right)+\Delta T\left(\alpha_{1}-\alpha_{0}\right)-\frac{1}{2} \varepsilon_{z, 1}^{h . e .} .
$$

Let us represent Eq. (18) in the form

$$
\frac{\partial^{2} \sigma_{x, 1}}{\partial x^{2}}-\omega^{2} \sigma_{x, 1}+\lambda^{2}=0
$$

where

$$
\left.\begin{array}{c}
\omega^{2}=\frac{2 g_{1}^{*}}{h_{1}}\left[\frac{\left(1-\mu_{1}\right)}{E_{1}}+\frac{h_{1}}{2 h_{0}} \frac{\left(1-\mu_{0}\right)}{E_{0}}\right]=g_{1}^{*}\left[\frac{2\left(1-\mu_{1}\right)}{E_{1} h_{1}}+\frac{\left(1-\mu_{0}\right)}{E_{0} h_{0}}\right], \\
\lambda^{2}=\frac{g_{1}^{*}}{h_{1}}\left[2 q\left(\frac{\mu_{1}}{E_{1}}-\frac{\mu_{0}}{E_{0}}\right)+2 \Delta T\left(\alpha_{0}-\alpha_{1}\right)+\varepsilon_{z, 1}^{h . e .}\right] .
\end{array}\right\}
$$

If at any time $\omega$ and $\lambda$ are known, the solution of equation (19) becomes the following expression:

$$
\sigma_{x, 1}=C_{1} e^{-\omega x}+C_{2} e^{\omega x}+\lambda^{2} / \omega^{2} \text {. }
$$

To find unknown constants $C_{1}$ and $C_{2}$ use the boundary conditions $\sigma_{x, 1}(l / 2)=\sigma_{x, 1}(-l / 2)=0$.

After the substitution boundary conditions in (21) we obtain:

$$
C_{1}=C_{2}=-\frac{\lambda^{2}}{\omega^{2}} \cdot \frac{1}{e^{\omega l / 2}+e^{-\omega l / 2}} \text {. }
$$

Thus, the final expression for the stresses has the form:

$$
\sigma_{x, 1}=\frac{\lambda^{2}}{\omega^{2}} \cdot\left(1-\frac{e^{-\omega x}+e^{\omega x}}{e^{\xi}+e^{-\xi}}\right)=\frac{\lambda^{2}}{\omega^{2}} \cdot\left[1-\frac{\cosh (\omega x)}{\cosh (\xi)}\right],
$$

where $\xi=\frac{\omega \cdot l}{2}$. From (6) follows

$$
\tau_{x z, 1}^{*}-\tau_{x z, 2}^{*}=h_{1} \frac{\partial \sigma_{x, 1}}{\partial x} .
$$

Given that $\tau_{x z, 2}^{*}=-\tau_{x z, 1}^{*}$ we get 


$$
\tau_{x z, 1}^{*}=-\frac{h_{1}}{2} \cdot \frac{\lambda^{2}}{\omega} \cdot \frac{\sinh (\omega x)}{\cosh (\xi)} .
$$

Let us assume that the model at the initial time has a temperature $T_{0}$. Then it cools to the test temperature $T_{1}$ with a speed $\beta_{T}$. After that the model is loaded by normal load at speed $\beta_{q}$ up to a certain value $q_{\max }$. Then, there is going the process of creep at $q=q_{\max }=$ const .

In the following results were used the next inputs:

- The geometric parameters of the model: $l=20 \mathrm{~mm}, h_{0}=10 \mathrm{~mm}, h_{1}=1 \mathrm{~mm}$

- The dependences the temperature of the model and the normal load on the time: $T(t)=\left\{\begin{array}{l}T_{0}-\beta_{T} t, t \leq\left(T_{0}-T_{1}\right) / \beta_{T} ; \\ T_{1}, t>\left(T_{0}-T_{1}\right) / \beta_{T} .\end{array} \quad q(t)=\left\{\begin{array}{l}0, t \leq\left(T_{0}-T_{1}\right) / \beta_{T} ; \\ \beta_{q}\left(t-\left(T_{0}-T_{1}\right) / \beta_{T}\right),\left(T_{0}-T_{1}\right) / \beta_{T}<t<\left(T_{0}-T_{1}\right) / \beta_{T}+q_{\max } / \beta_{q} ; \\ q_{\max }, t \geq\left(T_{0}-T_{1}\right) / \beta_{T}+q_{\max } / \beta_{q} .\end{array}\right.\right.$

- Elastic constants of the substrate:

$E_{0}=2 \cdot 10^{5} \mathrm{MPa}, \mu_{0}=0.33, \mu_{1}=0.37, g^{*}=9600 \mathrm{MPa} / \mathrm{mm}, \alpha_{0}=0.11 \cdot 10^{-4} \cdot 1 / \mathrm{K}$

- Dependences of the elastic and relaxation constants of the substrate and the adhesive on the temperature [2]:

$$
\begin{aligned}
& E_{\infty, 1,1}=\left\{\begin{array}{l}
\left(2,4 \cdot 10^{6} / T-6120\right) M \Pi a, T \leq 370 K ; \\
(2,23 T-458.6) M \Pi a, T>370 K ;
\end{array} \quad E_{\infty, 2,1}=0.1 E_{\infty, 1,1} ;\right. \\
& \eta_{0,1,1}=600 \cdot \exp \left(\frac{9500}{T}-21\right) \mathrm{MPa} \cdot \min ; \eta_{0,2,1}=600 \cdot \exp \left(\frac{35400}{T}-91.5\right) \mathrm{MPa} \cdot \min ; \\
& E_{1}=(-18.2 T+8200) \mathrm{MPa} ; \alpha_{1}=\left\{\begin{array}{l}
10^{-6}(0,46 T-58) 1 / K, T \leq 350 K ; \\
10^{-6}[102.69+110 \exp (-\exp (17.17-0.044 T))], T>350 K .
\end{array}\right.
\end{aligned}
$$

Fig. 5 shows a series of curves showing the change of tangential stresses in the contact layer near the edge of the sample $(x= \pm l / 2)$ with time under constant load $q_{\max }=20 \mathrm{M \Pi a}$, taking into account the residual stresses of temperature shrinkage. As can be seen from this figure, in the process of creep concentration of tangential stress increases with time, and increases the value of maximum stresses.

In the process of creep tangential stresses reach their asymptotic values. Fig. 6 shows the curves of changes maximum shear stresses for different values of $q_{\text {max }}$ excluding residual stresses of thermal shrinkage.

If we now choose a certain value $\tau_{a d}$ (the maximum

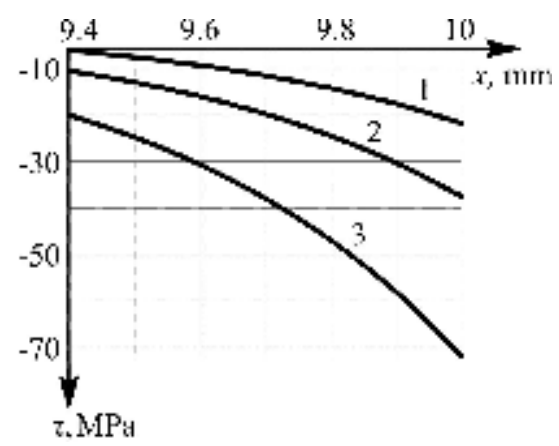

Fig. 5. The curves of $\tau_{x z, 1}^{*}$ at creep for different moments of time; 1 - $100 \mathrm{~min} ; 2-10^{5} \mathrm{~min} ; 3-10^{8} \mathrm{~min}$ allowable tangential stress in the contact layer), then we can construct curves of durability depending on the value of $q_{\max }$. Fig. 7 shows the curves for different values of $\tau_{a d}$ with account residual stresses of thermal shrinkage. 


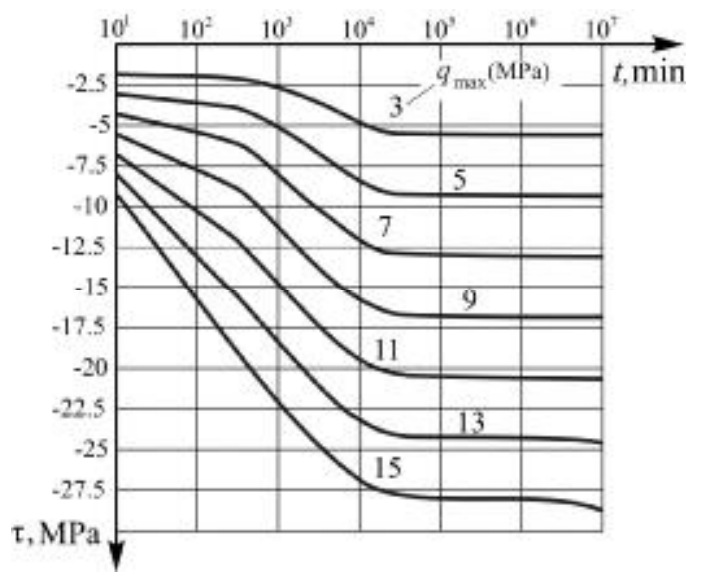

Fig. 6. Change of stresses (at $x= \pm l / 2$ ) with time for different values $q_{\max }(\mathrm{MPa})$

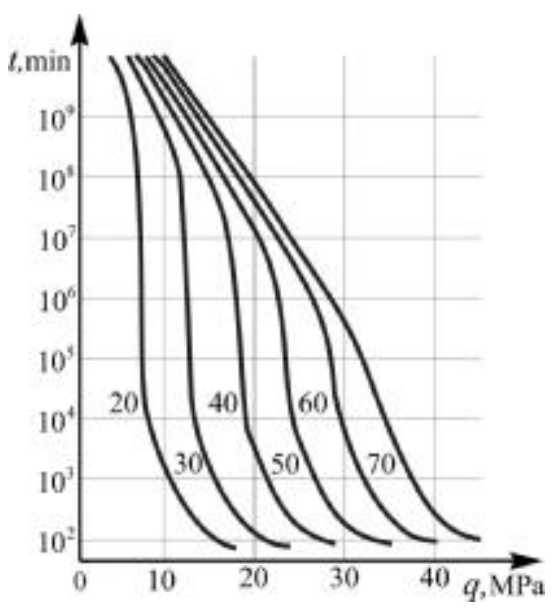

Fig. 7. Curves of durability layered composite for different values $\tau_{a d}(\mathrm{MPa})$

\section{Conclusions}

Based on obtained results, we can conclude that accounting for residual stresses of thermal shrinkage is required in the analysis of long-term strength of the layered composite because these stresses contribute significantly to the stress-strain state. Problems of strength of polymers and composites were considered by the authors in the works $[4,5]$.

\section{References}

[1] A.L. Rabinovich, Introduction to the mechanics of reinforced polymers, Nauka, Moscow, 1970. [2] R.A. Turusov, L.I. Manevich, Contact_Layer Method in Adhesive Mechanics: Adhesive Strength during Normal Detachment. Polymer Science, Series D. Glues and Sealing Materials. 3 No 1 (2010) 159-169.

[3] R.A.Turusov, L.I. Manevich, Determining Parameters of Rigidity and True Strength of an Adhesion Bond for a Contact Layer. Polymer Science, Series D. Glues and Sealing Materials. 4, No.1, (2011) $1-4$.

[4] R.A.Turusov, A. Kuperman, V.I. Andreev, Determining the True Strength of the Material of Fiberglass Thick Rings When Stretched with Half-disks, Advanced Materials Research. 1102 (2015) 155-159

[5] B.M. Yazyev, V.I. Andreev, R.A. Turusov, Some problems and methods of mechanics of macroinhomogenious viscoelastic medium, RGSU, Rostov-onDon, 2009. 